\title{
Role of strangeness to the neutron star mass and cooling
}

\author{
Chang-Hwan Lee ${ }^{1, \star}$, Yeunhwan Lim², Chang Ho Hyun ${ }^{3}$, and Kyujin Kwak ${ }^{4}$ \\ ${ }^{1}$ Department of Physics, Pusan National University, Busan 46241, Korea \\ ${ }^{2}$ Cyclotron Institute, Texas A\&M University, College Station, TX 77843, USA \\ ${ }^{3}$ Department of Physics Education, Daegu University, Gyeongsan 38453, Korea \\ ${ }^{4}$ Department of Physics, Ulsan National Institue of Science and Technology (UNIST), Ulsan 44919, Korea
}

\begin{abstract}
Neutron star provides unique environments for the investigation of the physics of extreme dense matter beyond normal nuclear saturation density. In such high density environments, hadrons with strange quarks are expected to play very important role in stabilizing the system. Kaons and hyperons are the lowest mass states with strangeness among meson and bayron families, respectively. In this work, we investigate the role of kaons and hyperons to the neutron star mass, and discuss their role in the neutron star cooling.
\end{abstract}

\section{Introduction}

Observations of two massive neutron stars $\left(\sim 2 M_{\odot}\right)$ in neutron star-white dwarf binaries raised questions on the role of strangeness [1, 2]. In general, for a given neutron star equation of state (EOS), strangeness softens the EOS and reduces the maximum mass compared to that without strangeness [3]. Before the observation of $2 M_{\odot}$ neutron stars, strangeness is often introduced to make soft EOS and reduce the maximum mass of neutron stars down to $1.5 M_{\odot}$ to be consistent with the observed neutron star masses in neutron star-neutron star binaries [3]. Due to the observation of $2 M_{\odot}$ neutron stars, the maximum mass of neutron star has to be bigger than $2 M_{\odot}$, and those soft EOSs with strangeness inconsistent with $2 M_{\odot}$ neutron stars are ruled out. However, the existence of strangeness in the central part of neutron stars still remains as an open problem.

In dense matter, as baryon number density increases, the energy densities of protons and neutrons increase because fermions cannot exist at the same states and higher energy states have to be included. Hence, it is quite natural for the new states with lower energy density to exist at high densities. Possible candidates for the new states are condensed pions, condensed kaons, hyperons, and deconfined quark states. In hadronic phase before the phase transition to the quark states, strangeness might appear as hadrons such as hyperons and kaons which are lowest energy states with strange quark (or strange anti-quark) among baryons and mesons, respectively.

In this work, based on the recent works by Lim et al. [4-7], we investigate the neutron star EOS with kaons and hyperons at the core, and check the effect of new EOS on the neutron star cooling. In Sec. 2, neutron star mass with strangeness is discussed. In Sec. 3, neutron star cooling with strangeness is discussed. Final conclusion is summarized in Sec. 4.

^e-mail: clee@pusan.ac.kr 


\section{Neutron star mass with strangeness}

In our works [4-6], in order to investigate the role of strangeness to neutron star EOS, we use Skyrme force models for $N N, N \Lambda$ and $\Lambda \Lambda$ interactions and $\mathrm{SU}(3)$ chiral effective models for the kaon-nucleon interactions. Even though strangeness reduces the maximum mass of neutron stars, we found that $2 M_{\odot}$ neutron star with strangeness is still possible if the original EOS without strangeness is stiff enough. In Table 1, we list the maximum mass of neutron stars with given EOS which are consistent with $2 M_{\odot}$ observations.

Table 1. Maximum mass of neutron stars with strangeness [4-6].

\begin{tabular}{|c|c|c|c|}
\hline \multicolumn{4}{|c|}{ with $\Lambda$ hyperons } \\
\hline$N N$ model & $N \Lambda$ model & $\Lambda \Lambda$ model & Maximum neutron star mass \\
\hline SKI4 & YBZ6 & $\mathrm{S} \Lambda \Lambda 3$ & $2.03 M_{\odot}$ \\
\hline SGI & YBZ6 & $\mathrm{S} \Lambda \Lambda 3$ & $2.04 M_{\odot}$ \\
\hline \multicolumn{4}{|c|}{ with kaon condensation } \\
\hline$N N$ model & $a_{3} m_{s}$ & $\Sigma_{K N}$ & Maximum neutron star mass \\
\hline SKI4 & $-134 \mathrm{MeV}$ & $167 \mathrm{MeV}$ & $2.06 M_{\odot}$ \\
\hline SGI & $-134 \mathrm{MeV}$ & $167 \mathrm{MeV}$ & $2.19 M_{\odot}$ \\
\hline SGI & $-178 \mathrm{MeV}$ & $256 \mathrm{MeV}$ & $2.03 M_{\odot}$ \\
\hline
\end{tabular}

For the contribution of $\Lambda$ hyperons, $\Lambda \Lambda$ interaction plays very important role. Without $\Lambda \Lambda$ interaction, EOS with $N \Lambda$ interaction becomes too soft to explain $2 M_{\odot}$ neutron stars. Hence, those models with repulsive $\Lambda \Lambda$ interaction are consistent with current observations. For the contribution of kaons, we found that the smaller values of $\Sigma_{K N}$ are consistent with observations. $\Sigma_{K N}$ is responsible for the attraction in the $K N$ scalar interaction and the reduction of the kaon effective mass in medium.

In summary, even though strangeness reduces the maximum mass of neutron stars, original stiffer nuclear EOS (without strangeness) combined with less attractive interactions for the strangeness can be consistent with $2 M_{\odot}$ neutron star observations.

\section{Neutron star cooling with strangeness}

In Lim et al. [7], we investigate the cooling behavior of neutron stars with strangeness for the selected models summarized in Sec. 2. In Figure 1, we show the cooling curves of neutron stars for SGI model without strangeness and SGI+YBZ6+S $\Lambda \Lambda 3$ model with hyperons [7]. In both cases, the sudden drop in temperature occurs in a very narrow mass range. These sudden drops are caused by the nucleon direct URCA. For SGI model, the direct URCA starts to occur when the neutron star mass reaches around $1.7-1.8 M_{\odot}$, and for SGI+YBZ6+S $\Lambda \Lambda 3$ model, the direct URCA starts to occur around $1.5-1.6 M_{\odot}$. So, the hyperons can have a significant effect on the neutron stars with masses around $1.5-1.8 M_{\odot}$ as far as cooling is concerned. Other models show similar behavior except for the shift in the mass ranges for sudden temperature drop.

For kaons, the cooling curves with and without kaon contribution are almost the same because the critical densities for kaon condensation are larger than the critical densities for direct URCA. This indicates that the kaon condensation occurs only for the neutron stars with masses higher than $1.8 M_{\odot}$ for SGI model. For SKI4 model, the critical mass for kaon condensation is higher than $1.7 M_{\odot}$. Note that the high critical mass for the kaon condensation results in the high maximum mass of neutron star as in Table 1. 

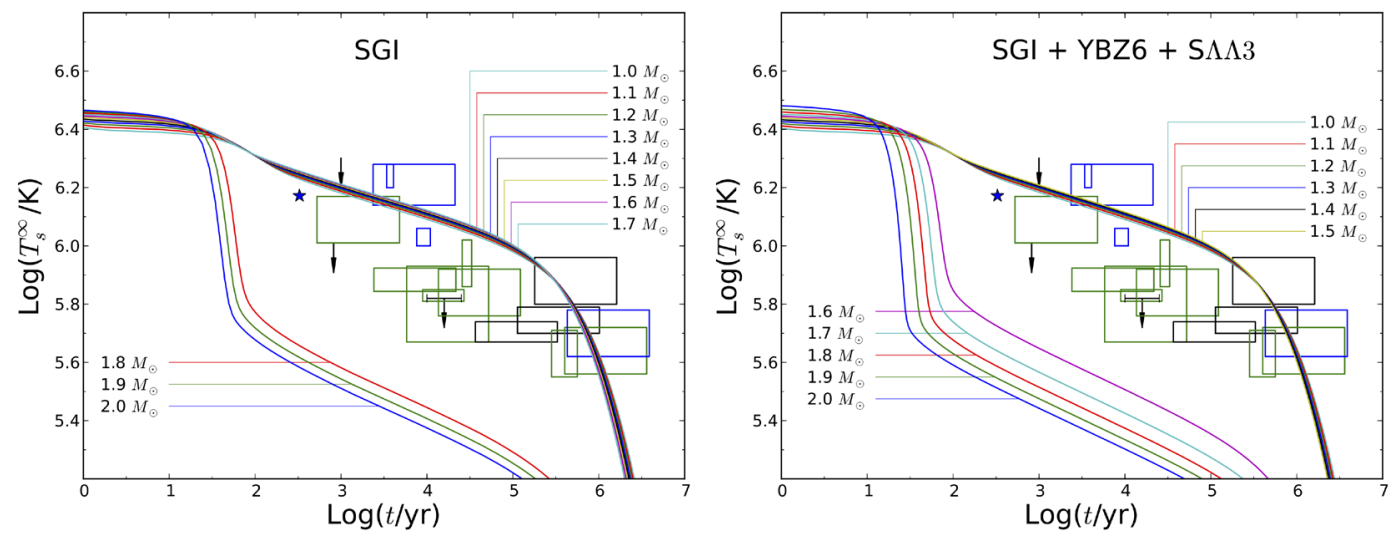

Figure 1. Cooling curves of neutron stars (taken from Lim et al. [7]). Left figure corresponds to SGI model (without strangeness) and right figure to SGI+YBZ6+S $\Lambda$ 3 model (with hyperons). Cooling curves with SGI model combined with kaon interaction are almost the same as in the left figure.

The sudden drop of the cooling curves in a very narrow mass range in Figure 1 raises a question on the mass distribution function of neutron stars because masses of observed neutron stars are distributed in a wider mass range [3].

\section{Discussion}

In this article, we discuss the role of strangeness to the maximum mass and cooling behavior of neutron stars. Even though particles with strangeness such as hyperons and kaons soften the EOS and consequently reduce the maximum mass of neutron stars, the specific value of maximum mass strongly depends on the original (without strangeness) nuclear EOS. If the original EOS is stiff enough, even with strangeness, the maximum mass can still be consistent with the $2 M_{\odot}$ neutron star observations $[1,2]$. Among Skyrme force models, SKI4 and SGI models satisfy these constraints [4-6]. We found that the repulsive $\Lambda \Lambda$ interaction $(\mathrm{S} \Lambda \Lambda 3)$ and less attractive $K N$ interaction (corresponding to the smaller $\Sigma_{K} N$ ) can be consistent with $2 M_{\odot}$ neutron star observations.

Using these models, we investigate the cooling behavior of neutron stars. With original (without strangeness) SKI4 and SGI nuclear models, the temperature drops very rapidly as soon as the direct URCA occurs. The sudden drop of the cooling curves in the left panel of Figure 1 occurs for a very narrow mass range around $1.7-1.8 M_{\odot}$. This behavior remains the same in the right panel except for the shift in mass range around $1.5-1.6 M_{\odot}$. The sudden drop of the cooling curve in a very narrow mass range raises a question on the mass distribution, because it may not be consistent with the mass distribution of well-measured neutron star masses [3]. Since our work is focused on the Skyrme force models, we cannot make a firm conclusion. However, we confirm that the strangeness in neutron star is still an open possibility.

Many new developments in the neutron star research have been in progress. First of all, analyses on the low-mass X-ray binaries (LMXBs) have provided constraints on both masses and radii of neutron stars even though the uncertainty is large [8, 9]. New X-ray mission NICER (The Neutron star Interior Composition ExploreR) was launched in June 2017. In near future, NICER is expected to provide new observational data on the neutron stars in LMXBs [10]. Gravitational wave detection 
from neutron star binaries is another possibility. After the first success of the detection of gravitational waves from black hole binaries [11-13], gravitational waves generated by neutron star-neutron star or neutron star-black hole binaries are expected to be detected in near future. In the merger process, the inner structure of neutron star can be revealed via gravitational waves [14].

\section{Acknowledgments}

This article is based on the talk given at the International Conference on Gravitation: Joint Conference of ICGAC-XIII and IK15, July 3-7, 2017, Ewha Womans University, Seoul, Korea. CHL is partially supported by the National Research Foundation of Korea (NRF) grant funded by the Korea government (MSIP) No. 2015R1A2A2A01004238. CHL and KK are partially supported by the NRF grant No. 2016R1A5A1013277.

\section{References}

[1] P. Demorest, T. Pennucci, S. Ransom, M. Roberts, and J. Hessels, Nature 467, 1081 (2010).

[2] J. Antoniadis et al., Science 340, 448 (2013).

[3] M. Prakash, Proceedings of 8th International Workshop on Critical Point and Onset of Deconfinement, arXiv:1307.0397 (2013).

[4] Y. Lim, K. Kwak, C. H. Hyun, C.-H. Lee, Phys. Rev. C 89, 055804 (2014).

[5] Y. Lim, C. H. Hyun, K. Kwak, C.-H. Lee, Int. J. of Mod. Phys. E 24, 1550100 (2015).

[6] Y. Lim, C. H. Hyun, C.-H. Lee, Int. J. of Mod. Phys. E 26, 1750015 (2017).

[7] Y. Lim, C. H. Hyun, C.-H. Lee, Strangeness in neutron star cooling, arXiv:1608.02078.

[8] A. W. Steiner, J. M. Lattimer, and E. F. Brown, Astrophys. J. 722, 33 (2010).

[9] A. W. Steiner, J. M. Lattimer, and E. F. Brown, Eur. Phys. J. A 52, 18 (2016).

[10] K. C. Gendreau, et al., "The Neutron star Interior Composition ExploreR (NICER): Design and Development", Proc. SPIE 9905, Space Telescopes and Instrumentation 2016; Ultraviolet to Gamma Ray, 99051H (22 July 2016).

[11] B. P. Abbott et al. (LIGO Scientific and Virgo Collaboration), Phys. Rev. Lett.116, 061102 (2016).

[12] B. P. Abbott et al. (LIGO Scientific and Virgo Collaboration), Phys. Rev. Lett. 116, 241103 (2016).

[13] B. P. Abbott et al. (LIGO Scientific and Virgo Collaboration), Phys. Rev. Lett. 118, 221101 (2017).

[14] K. Kiuchi, Y. Sekiguchi, K. Kyutoku, and M. Shibata, Classical and Quantum Gravity 29, 124003 (2012). 